\section{ER-028 PERCEPTION OF HOSPITAL PHARMACISTS TOWARDS PHARMACOGENETIC TESTING}

AM Xuereb*, F Wirth, A Serracino-Inglott. Department of Pharmacy, Faculty of Medicine and Surgery- University of Malta, Msida, Malta

10.1136/ejhpharm-2019-eahpconf.625

Background Advances in pharmacogenetics provide the potential for expansion of the role of hospital pharmacists in personalised medicine.

Purpose To assess the awareness, attitudes and confidence of hospital pharmacists regarding pharmacogenetic testing.

Material and methods An anonymous self-administered questionnaire was developed, validated and tested for reliability. An online version of the questionnaire was created using SurveyMonkey and was disseminated via electronic mail after ethics approval to 70 hospital pharmacists practicing in four hospitals (three public and one private). Descriptive statistics were calculated for the responses received.

Results Forty-two hospital pharmacists (24 females, 18 males, age range 21-55 years) completed the questionnaire. Fortyone pharmacists were aware of the term 'pharmacogenetic testing'. Pharmacists agreed that pharmacogenetic testing: guides individualised therapy selection and dosing $(n=41)$; is useful in cases of treatment-resistance $(n=39)$ and intolerance $(n=36)$; should be a government-funded service $(n=30)$; should be routinely implemented for medication therapy management $(n=25)$; leads to reduced healthcare costs $(n=24)$; and is applicable for use in their practice $(n=21)$. Twenty-one pharmacists perceived oncology drugs as the drugs for which pharmacogenetic testing is most applicable. The challenges of pharmacogenetic testing perceived by the pharmacists were: cost issues $(n=41)$; lack of healthcare, professional and public awareness $(n=39)$; increased waiting time for clinical actions by prescribers $(n=29)$; and ethical concerns $(n=26)$.

Seventeen pharmacists encountered the need to order a pharmacogenetic test at least once monthly, but none of them had ever ordered a test. The pharmacists expressed a lack of confidence in recommending $(n=31)$ and ordering $(n=30)$ a pharmacogenetic test when indicated, in interpreting test results $(n=35)$ and in discussing test results with physicians and patients $(\mathrm{n}=31)$. Thirty-eight pharmacists agreed that they required more education on pharmacogenetics to increase competency and confidence. Seminars $(n=29)$ and courses $(n=24)$ were the preferred approaches for further education.

Conclusion Hospital pharmacists in this study were aware of pharmacogenetic testing and recognised its benefits, applicability and challenges. The pharmacists expressed a lack of confidence in the practical aspects of pharmacogenetic testing and were in agreement regarding the need for further education on the subject.

\section{REFERENCES AND/OR ACKNOWLEDGEMENTS}

N/A.

No conflict of interest. 